\title{
ON A BERRY-ESSEEN TYPE BOUND FOR THE MAXIMUM LIKELIHOOD ESTIMATOR OF A PARAMETER FOR SOME STOCHASTIC PARTIAL DIFFERENTIAL EQUATIONS
}

\author{
M. N. MISHRA AND B. L. S. PRAKASA RAO \\ Received 24 September 2003 and in revised form 11 March 2004
}

This paper is concerned with the study of the rate of convergence of the distribution of the maximum likelihood estimator of a parameter appearing linearly in the drift coefficients of two types of stochastic partial differential equations (SPDEs).

\section{Introduction}

Maximum likelihood estimation of a parameter appearing linearly in some stochastic partial differential equations (SPDEs) has been considered by Hübner et al. [3]. Detailed discussion of these SPDEs and some interesting phenomena arising out of the parameter estimation have been considered by them in two examples. In this paper, we study the rate of convergence of the distribution of the maximum likelihood estimator (MLE) $\hat{\theta}_{N, \epsilon}$ of the parameter $\theta$ occurring linearly in such SPDEs. Bounds on the difference $\left|\hat{\theta}_{N, \epsilon}-\theta_{0}\right|$, where $\theta_{0}$ is the true value of the parameter, can be obtained using these results as in Mishra and Prakasa Rao [6]. In Section 2, we describe a SPDE with parameter $\theta$ such that the corresponding stochastic process $u_{\epsilon}$ generates measures $\left\{P_{\theta}^{\epsilon}, \theta \in \Theta\right\}$ which are mutually absolutely continuous, and the main results pertaining to this section have been described in Section 3. In Section 4, we describe a SPDE with parameter $\theta$ such that the corresponding stochastic process $u_{\epsilon}$ generates measures which form a family of probability measures $\left\{P_{\theta}^{\epsilon}, \theta \in \Theta\right\}$ which are singular with respect to each other, and this section also contains the main results connected to this problem. Comprehensive surveys on statistical inference for such classes of SPDEs are given by Prakasa Rao [7, 8]. Throughout the paper, we will denote by $C$ a positive constant different at different places of occurrence, possibly dependent on the initial conditions of the SPDEs.

\section{SPDE with linear drift (absolutely continuous case): estimation}

Let $(\Omega, \mathscr{F}, P)$ be a probability space and consider the process $u_{\epsilon}(t, x), 0 \leq x \leq 1,0 \leq t \leq T$, governed by the SPDE

$$
d u_{\epsilon}(t, x)=\left(\Delta u_{\epsilon}(t, x)+\theta u_{\epsilon}(t, x)\right) d t+\epsilon d W_{Q}(t, x)
$$

Copyright (C) 2004 Hindawi Publishing Corporation Journal of Applied Mathematics and Stochastic Analysis 2004:2 (2004) 109-122 2000 Mathematics Subject Classification: 62M40, 60H15

URL: http://dx.doi.org/10.1155/S1048953304309019 
with the initial and boundary conditions given by

$$
\begin{aligned}
& u_{\epsilon}(0, x)=f(x), \quad f \in L_{2}[0,1], \\
& u_{\epsilon}(t, 0)=u_{\epsilon}(t, 1)=0, \quad 0 \leq t \leq T,
\end{aligned}
$$

where $\Delta=\partial^{2} / \partial x^{2}$. Let $\epsilon \rightarrow 0$ and $\theta \subset \mathbb{R}$. Here, $Q$ is a nuclear covariance operator for the Wiener process $W_{Q}(t, x)$ taking values in $L_{2}[0,1]$ so that $W_{Q}(t, x)=Q^{1 / 2} W(t, x)$ and $W(t, x)$ is a cylindrical Brownian motion in $L_{2}[0,1]$. Then it is known that (cf. Rozovskiu [9])

$$
W_{Q}(t, x)=\sum_{i=1}^{\infty} q_{i}^{1 / 2} e_{i}(x) W_{i}(t) \quad \text { a.s. }
$$

where $\left\{W_{i}(t), 0 \leq t \leq T\right\}, i \geq 1$, are independent one-dimensional standard Wiener processes, $\left\{e_{i}\right\}$ is a complete orthonormal system (CONS) in $L_{2}[0,1]$ consisting of the eigenvectors of $Q$, and $\left\{q_{i}\right\}$ are the corresponding eigenvalues of $Q$. We consider a special covariance operator $Q$ with $e_{k}=\sin k \pi x, k \geq 1$, and $\lambda_{k}=(\pi k)^{2}, k \geq 1$. Then $\left\{e_{n}\right\}$ is a CONS with the eigenvalues $q_{i}=\left(1+\lambda_{i}\right)^{-1}, i \geq 1$, for the operator $Q$, where $Q=(I-\Delta)^{-1}$. Furthermore, $d W_{Q}=Q^{1 / 2} d W$. We define a solution $u_{\epsilon}(t, x)$ of $(2.1)$ as a formal sum:

$$
u_{\epsilon}(t, x)=\sum_{i=1}^{\infty} u_{i \epsilon}(t) e_{i}(x)
$$

(cf. Rozovskir [9]). It is known that the Fourier coefficients $u_{i \epsilon}(t)$ satisfy the stochastic differential equation

$$
d u_{i \epsilon}(t)=\left(\theta-\lambda_{i}\right) u_{i \epsilon}(t) d t+\frac{\epsilon}{\sqrt{\lambda_{i}+1}} d W_{i}(t), \quad 0<t \leq T,
$$

with the initial conditions

$$
u_{i \epsilon}(0)=v_{i}, \quad v_{i}=\int_{0}^{1} f(x) e_{i}(x) d x .
$$

It is further known that the function $u_{\epsilon}(t, x)$ as defined above belongs to $L_{2}([0, T] \times$ $\left.\Omega ; L_{2}[0,1]\right)$ together with its derivative in $t$. Furthermore, $u_{\epsilon}(t, x)$ is the only solution of (2.1) under the boundary conditions (2.2) and (2.3). Let $P_{\theta}^{\epsilon}$ be the measure generated by $u_{\epsilon}$ on $C[0, T]$ when $\theta$ is the true parameter. It has been shown by Hübner et al. [3] that the family of measures $\left\{P_{\theta}^{(\epsilon)}, \theta \in \Theta\right\}$ is mutually absolutely continuous and

$$
\begin{aligned}
\log \frac{d P_{\theta}^{\epsilon}}{d P_{\theta_{0}}}\left(u_{\epsilon}\right)=\sum_{i=1}^{\infty} \frac{\lambda_{i}+1}{\epsilon^{2}}[ & \left(\theta-\theta_{0}\right) \int_{0}^{T} u_{i \epsilon}(t) d u_{i \epsilon}(t) \\
& \left.-\frac{1}{2}\left\{\left(\theta-\lambda_{i}\right)^{2}-\left(\theta_{0}-\lambda_{i}\right)^{2}\right\} \int_{0}^{T} u_{i \epsilon}^{2}(t) d t\right] .
\end{aligned}
$$

The projection of the solution $u_{\epsilon}(t, x)$ onto the subspace $\pi^{N}$ spanned by $\left\{e_{1}, e_{2}, \ldots, e_{N}\right\}$ (see Liptser and Shiryayev [4]) is given by $u_{\epsilon}^{N}(t, x)=\sum_{i=1}^{N} u_{i \epsilon}(t) e_{i}(x)$. Let $P_{\theta}^{\epsilon, N}$ be the probability measure generated by the process $u_{\epsilon}^{N}(t, x)$ on $C[0, T]$ when $\theta$ is the true parameter. 
Then the measure $P_{\theta}^{\epsilon, N}$ is absolutely continuous with respect to the measure $P_{\theta_{0}}^{\epsilon, N}$ and $\log \frac{d P_{\theta}^{\epsilon, N}}{d P_{\theta_{0}}^{\epsilon, N}}=\sum_{i=1}^{N} \frac{\lambda_{i}+1}{\epsilon^{2}}\left[\left(\theta-\theta_{0}\right) \int_{0}^{T} u_{i \epsilon}(t) d u_{i \epsilon}(t)-\frac{1}{2}\left\{\left(\theta-\lambda_{i}\right)^{2}-\left(\theta_{0}-\lambda_{i}\right)^{2}\right\} \int_{0}^{T} u_{i \epsilon}(t) d t\right]$.

The MLE of the parameter $\theta$ is given by

$$
\hat{\theta}_{N, \epsilon}=\frac{\sum_{i=1}^{N}\left(\lambda_{i}+1\right) \int_{0}^{T} u_{i \epsilon}(t)\left(d u_{i \epsilon}(t)+\lambda_{i} u_{i \epsilon}(t) d t\right)}{\sum_{i=1}^{N}\left(\lambda_{i}+1\right) \int_{0}^{T} u_{i \epsilon}^{2}(t) d t}
$$

(cf. [3, page 154]).

\section{SPDE with linear drift (absolutely continuous case): Berry-Esseen type bound}

We now prove two theorems leading to a Berry-Esseen type bound for the MLE $\hat{\theta}_{N, \epsilon}$. It can be checked that $E_{\theta_{0}} \int_{0}^{T} u_{i \epsilon}^{2}(t) d t<\infty$ for $i \geq 1$. We assume that $\theta_{0}<\pi^{2}$, where $\theta_{0}$ is the true parameter. Let $\Phi(\cdot)$ denote the standard normal distribution function and define

$$
Q_{N, T}^{(\epsilon)}=\sum_{i=1}^{N} \frac{\lambda_{i}+1}{2\left(\theta-\lambda_{i}\right)}\left(v_{i}^{2}\left(e^{2\left(\theta-\lambda_{i}\right) T}-1\right)-T \frac{\epsilon^{2}}{\lambda_{i}+1}\right) .
$$

Theorem 3.1. For any $0<\delta<1$,

$$
\begin{aligned}
\sup _{y} \mid & P_{\theta_{0}}^{\epsilon, N}\left\{\sqrt{Q_{N, T}^{(\epsilon)}} \epsilon^{-1}\left(\hat{\theta}_{N, \epsilon}-\theta_{0}\right) \leq y\right\}-\Phi(y) \mid \\
& \leq 2 P_{\theta_{0}}^{\epsilon, N}\left\{\left|\sum_{i=1}^{N} \frac{\lambda_{i}+1}{Q_{N, T}^{(\epsilon)}} \int_{0}^{T} u_{i \epsilon}^{2}(t) d t-1\right| \geq \delta\right\}+3 \sqrt{\delta} .
\end{aligned}
$$

Theorem 3.2. Let $N \geq 1$ be fixed. Then there exists a constant $C$ depending on $\theta_{0},\|f\|$, and $T$ such that, for any $0<\delta \leq 1$ and $0<\epsilon<1$,

$$
P_{\theta_{0}}^{\epsilon, N}\left\{\left|\sum_{i=1}^{N} \frac{\lambda_{i}+1}{Q_{N, T}^{(\epsilon)}} \int_{0}^{T} u_{i \epsilon}^{2}(t) d t-1\right| \geq \delta\right\} \leq C \frac{\epsilon}{\delta}\left(\frac{1+T^{1 / 2}}{Q_{N, T}^{(\epsilon)}}\right) .
$$

We first state two lemmas needed in the sequel.

Lemma 3.3. Let $(\Omega, \mathscr{F}, P)$ be a probability space and let $f$ and $g$ be $\mathscr{F}$-measurable functions. Then, for any $\delta>0$,

$$
\begin{aligned}
\sup _{x} \mid & P\left\{\omega: \frac{f(\omega)}{g(\omega)} \leq x\right\}-\Phi(x) \mid \\
& \leq \sup _{y}|P\{\omega: f(\omega) \leq y\}-\Phi(y)|+P\{\omega:|g(\omega)-1| \geq \delta\}+\delta .
\end{aligned}
$$

Proof. See Michel and Pfanzagl [5]. 


\section{Berry-Esseen type bound for SPDE}

Lemma 3.4. Let $\{W(t), t \geq 0\}$ be a standard Wiener process and let $Z$ be a nonnegative random variable. Then, for every $x \in \mathbb{R}$ and $\delta>0$,

$$
|P\{W(Z) \leq x\}-\Phi(x)| \leq(2 \delta)^{1 / 2}+P\{|Z-1| \geq \delta\} .
$$

Proof. See Hall and Heyde [2, page 85].

Proof of Theorem 3.1. It follows from (2.10) that

$$
\sqrt{Q_{N, T}^{(\epsilon)}} \epsilon^{-1}\left(\hat{\theta}_{N, \epsilon}-\theta_{0}\right)=\frac{\left\{\sum_{i=1}^{N} \sqrt{\lambda_{i}+1} \int_{0}^{1} u_{i \epsilon}(t) d W_{i}(t)\right\} / \sqrt{Q_{N, T}^{(\epsilon)}}}{\left\{\sum_{i=1}^{N}\left(\lambda_{i}+1\right) \int_{0}^{T} u_{i \epsilon}^{2}(t) d t\right\} / Q_{N, T}^{(\epsilon)}} .
$$

Now, for any $y \in \mathbb{R}$,

$$
\begin{aligned}
\mid P_{\theta_{0}}^{\epsilon, N}\{ & \left.\sqrt{Q_{N, T}^{(\epsilon)}} \epsilon^{-1}\left(\hat{\theta}_{N, \epsilon}-\theta_{0}\right) \leq y\right\}-\Phi(y) \mid \\
\leq & \left|P_{\theta_{0}}^{\epsilon, N}\left\{\frac{\sum_{i=1}^{N} \sqrt{\lambda_{i}+1} \int_{0}^{T} u_{i \epsilon}(t) d W_{i}(t) / \sqrt{Q_{N, \epsilon}^{(\epsilon)}}}{\sum_{i=1}^{N}\left(\lambda_{i}+1\right) \int_{0}^{T} u_{i \epsilon}^{2}(t) d t / Q_{N, T}^{(\epsilon)}} \leq y\right\}-\Phi(y)\right| \\
\leq & \sup _{x}\left|P_{\theta_{0}}^{\epsilon, N}\left\{\frac{\sum_{i=1}^{N} \sqrt{\lambda_{i}+1} \int_{0}^{T} u_{i \epsilon}(t) d W_{i}(t)}{\sqrt{Q_{N, T}^{(\epsilon)}}} \leq x\right\}-\Phi(x)\right| \\
& +P_{\theta_{0}}^{\epsilon, N}\left\{\left|\frac{\sum_{i=1}^{N}\left(\lambda_{i}+1\right) \int_{0}^{T} u_{i, \epsilon}^{2}(t) d t}{Q_{N, T}^{(\epsilon)}}-1\right| \geq \delta\right\}+\delta \quad \text { (by Lemma 3.3) } \\
= & \sup _{x}\left|P_{\theta_{0}}^{\epsilon, N}\left\{\widetilde{W}\left(\sum_{i=1}^{N} \frac{\lambda_{i}+1}{Q_{N, T}^{(\epsilon)}} \int_{0}^{T} u_{i \epsilon}^{2}(t) d t\right) \leq x\right\}-\Phi(x)\right| \\
& +P_{\theta_{0}}^{\epsilon, N}\left\{\left|\sum_{i=1}^{N} \frac{\lambda_{i}+1}{Q_{N, T}^{(\epsilon)}} \int_{0}^{T} u_{i \epsilon}^{2}(t) d t-1\right| \geq \delta\right\}+\delta,
\end{aligned}
$$

where $\widetilde{W}(\cdot)$ is an independent standard Wiener process by using Theorem 2.3 in Feigin [1] (due to Kunita-Watanabe) and the fact that $\int_{0}^{T} u_{i \epsilon}^{2}(t) d W_{i}(t), 1 \leq i \leq n$, are independent square-integrable martingales.

Hence

$$
\begin{aligned}
& \left|P_{\theta_{0}}^{\epsilon, N}\left\{\sqrt{Q_{N, T}^{(\epsilon)}} \epsilon^{-1}\left(\hat{\theta}_{N, \epsilon}-\theta_{0}\right) \leq y\right\}-\Phi(y)\right| \\
& \quad \leq \sqrt{2 \delta}+2 P_{\theta_{0}}^{\epsilon, N}\left\{\left|\sum_{i=1}^{N} \frac{\lambda_{i}+1}{Q_{N, T}^{(\epsilon)}} \int_{0}^{T} u_{i \epsilon}^{2}(t) d t-1\right| \geq \delta\right\}+\delta \quad \text { (by Lemma 3.4) } \\
& \quad \leq 2\left[P_{\theta_{0}}^{\epsilon, N}\left\{\left|\sum_{i=1}^{N} \frac{\lambda_{i}+1}{Q_{N, T}^{(\epsilon)}} \int_{0}^{T} u_{i \epsilon}^{2}(t) d t-1\right| \geq \delta\right\}\right]+3 \sqrt{\delta}
\end{aligned}
$$

for $0<\delta \leq 1$. 
Proof of Theorem 3.2. From (2.6), we obtain that

$$
\begin{gathered}
d u_{i \epsilon}(s)=\left(\theta-\lambda_{i}\right) u_{i \epsilon}(s) d s+\frac{\epsilon}{\sqrt{\lambda_{1}+1}} d W_{i}(s), \quad 0<t \leq T, \\
u_{i \epsilon}(0)=v_{i} .
\end{gathered}
$$

By the Îto formula, we have

$$
d\left(u_{i \epsilon}(s) \bar{e}^{\left(\theta-\lambda_{i}\right) s}\right)=\frac{\epsilon}{\sqrt{\lambda_{i}+1}} \bar{e}^{-\left(\theta-\lambda_{i}\right) s} d W_{i}(s)
$$

or

$$
u_{i \epsilon}(t) \bar{e}^{\left(\theta-\lambda_{i}\right) t}-v_{i}=\int_{0}^{t} \frac{\epsilon}{\sqrt{\lambda_{i}+1}} \bar{e}^{\left(\theta-\lambda_{i}\right) s} d W_{i}(s)
$$

Furthermore,

$$
d\left(u_{i \epsilon}^{2}(t)\right)=2\left(\theta-\lambda_{i}\right) u_{i \epsilon}^{2}(t) d t+\frac{\epsilon}{\sqrt{\lambda_{i}+1}} u_{i \epsilon}(t) d W_{i}(t)+\frac{\epsilon^{2}}{\lambda_{i}+1} d t
$$

or, equivalently,

$$
\begin{aligned}
& \frac{\lambda_{i}+1}{2\left(\theta-\lambda_{i}\right)} u_{i \epsilon}^{2}(T)-\frac{\lambda_{i}+1}{2\left(\theta-\lambda_{i}\right)} v_{i}^{2} \\
& \quad=\int_{0}^{T}\left(\lambda_{i}+1\right) u_{i \epsilon}^{2}(t) d t+\frac{\epsilon \sqrt{\lambda_{i}+1}}{2\left(\theta-\lambda_{i}\right)} \int_{0}^{T} u_{i \epsilon}(t) d W_{i}(t)+\frac{\epsilon^{2}}{2\left(\theta-\lambda_{i}\right)} T .
\end{aligned}
$$

We know, from (3.11), that

$$
\begin{aligned}
u_{i \epsilon}^{2}(T)= & v_{i}^{2} e^{2\left(\theta-\lambda_{i}\right) T}+e^{2\left(\theta-\lambda_{i}\right) T}\left(\frac{\epsilon}{\sqrt{\lambda_{i}+1}} \int_{0}^{T} \bar{e}^{\left(\theta-\lambda_{i}\right) s} d W_{i}(s)\right)^{2} \\
& +2 v_{i} e^{2\left(\theta-\lambda_{i}\right) T} \int_{0}^{T} \frac{\epsilon}{\sqrt{\lambda_{i}+1}} \bar{e}^{-\left(\theta-\lambda_{i}\right) s} d W_{i}(s)
\end{aligned}
$$

From (3.11) and (3.13), we obtain that

$$
\begin{gathered}
\sum_{i=1}^{N} \frac{\lambda_{i}+1}{2\left(\theta-\lambda_{i}\right)}\left\{v_{i}^{2}\left(e^{2\left(\theta-\lambda_{i}\right) T}-1\right)-\frac{\epsilon^{2}}{\lambda_{i}+1} T\right\}-\sum_{i=1}^{N} \int_{0}^{T}\left(\lambda_{i}+1\right) u_{i \epsilon}^{2}(t) d t \\
=\sum_{i=1}^{N} \frac{\epsilon \sqrt{\lambda_{i}+1}}{2\left(\theta-\lambda_{i}\right)} \int_{0}^{T} u_{i \epsilon}(t) d W_{i}(t) \\
-\sum_{i=1}^{N} \frac{\lambda_{i}+1}{2\left(\theta-\lambda_{i}\right)}\left[e^{2\left(\theta-\lambda_{i}\right) T}\left(\frac{\epsilon}{\sqrt{\lambda_{i}+1}} \int_{0}^{T} \bar{e}^{\left(\theta-\lambda_{i}\right) s} d W_{i}(s)\right)^{2}\right. \\
\left.-\frac{2 v_{i} \epsilon}{\sqrt{\lambda_{i}+1}} e^{2\left(\theta-\lambda_{i}\right) T} \int_{0}^{T} \bar{e}^{\left(\theta-\lambda_{i}\right) s} d W_{i}(s)\right] .
\end{gathered}
$$


114 Berry-Esseen type bound for SPDE

Since

$$
Q_{N, T}^{(\epsilon)}=\sum_{i=1}^{N} \frac{\lambda_{i}+1}{2\left(\theta-\lambda_{i}\right)}\left(v_{i}^{2}\left(e^{2\left(\theta-\lambda_{i}\right) T}-1\right)-T \frac{\epsilon^{2}}{\lambda_{i}+1}\right),
$$

we have

$$
\begin{aligned}
P_{\theta}^{\epsilon, N}\{\mid & \left.\frac{\int_{0}^{T}\left(\sum_{i=1}^{N}\left(\lambda_{i}+1\right) u_{i \epsilon}^{2}(t)\right) d t}{Q_{N, T}^{(\epsilon)}}-1 \mid \geq \delta\right\} \\
\leq & P_{\theta}^{\epsilon, N}\left\{\left|\sum_{i=1}^{N} \frac{\left(\epsilon \sqrt{\lambda_{i}+1} / 2\left(\theta-\lambda_{i}\right)\right) \int_{0}^{T} u_{i \epsilon}(t) d W_{i}(t)}{Q_{N, T}^{(\epsilon)}}\right| \geq \frac{\delta}{3}\right\} \\
& +P_{\theta_{0}}^{\epsilon, N}\left\{\left|\frac{\sum_{i=1}^{N}\left(2 v_{i} \epsilon / \sqrt{\lambda_{i}+1}\right) e^{2\left(\theta-\lambda_{i}\right) T} \int_{0}^{T} \bar{e}^{\left(\theta-\lambda_{i}\right) s} d W_{i}(s)}{Q_{N, T}^{(\epsilon)}}\right| \geq \frac{\delta}{3}\right\} \\
& +P_{\theta}^{\epsilon, N}\left\{\left|\frac{\sum_{i=1}^{N}\left(\epsilon^{2} / 2\left(\theta-\lambda_{i}\right)\right) e^{2\left(\theta-\lambda_{i}\right) T}\left(\int_{0}^{T} \bar{e}^{\left(\theta-\lambda_{i}\right) s} d w_{i}(s)\right)^{2}}{Q_{N, T}^{(\epsilon)}}\right| \geq \frac{\delta}{3}\right\} \\
= & I_{1}+I_{2}+I_{3} \text { (say). }
\end{aligned}
$$

Now

$$
\begin{aligned}
& I_{1} \leq \frac{C \epsilon}{\delta Q_{N, T}^{(\epsilon)}}\left\{\sum_{k=1}^{N} \frac{\lambda_{k}+1}{\left(\theta-\lambda_{k}\right)^{2}} E_{\theta_{0}} \int_{0}^{T} u_{k \epsilon}^{2}(t) d t\right\}^{1 / 2} \\
& \leq \frac{C \epsilon}{\delta Q_{N, T}^{(\epsilon)}}\left\{\sum_{k=1}^{N} \frac{\lambda_{k}+1}{\left(\theta-\lambda_{k}\right)^{2}}\left(\frac{1}{2\left(\theta-\lambda_{k}\right)} v_{k}^{2}\left(1-e^{2\left(\theta-\lambda_{k}\right) T}\right)\right)\right. \\
&\left.+\frac{\epsilon^{2}}{2} \frac{1}{\left(\lambda_{k}-\theta\right)^{3}}\left(T-\frac{1-\bar{e}^{2\left(\lambda_{k}-\theta\right) T}}{2\left(\lambda_{k}-\theta\right)}\right)\right\}^{1 / 2}
\end{aligned}
$$

(following [3, page 154])

$$
\begin{aligned}
& \leq \frac{C \epsilon}{\delta Q_{N, T}^{(\epsilon)}} \sum_{k=1}^{N}\left\{\frac{\lambda_{k}+1}{\left(\lambda_{k}-\theta\right)^{3}} v_{k}^{2}\left(1-\bar{e}^{2\left(\lambda_{k}-\theta\right) T}\right)+\frac{\epsilon^{2}}{2} \frac{T}{\left(\lambda_{k}-\theta\right)^{3}}\right\}^{1 / 2} \\
& \leq \frac{C \epsilon}{\delta Q_{N, T}^{(\epsilon)}} \sum_{k=1}^{N}\left\{\frac{\lambda_{k}+1}{\left(\lambda_{k}-\theta\right)^{3}} v_{k}^{2}+\frac{\epsilon^{2} T}{\left(\lambda_{k}-\theta\right)^{3}}\right\}^{1 / 2} \\
& \leq \frac{C \epsilon}{\delta Q_{N, T}^{(\epsilon)}}\left\{\sum_{k=1}^{N} \frac{\|f\|}{k^{2}}+\epsilon T^{1 / 2} \sum_{k=1}^{N} \frac{1}{k^{3}}\right\} \\
& \leq \frac{C \epsilon}{\delta Q_{N, T}^{(\epsilon)}}+\frac{C \epsilon T^{1 / 2}}{\delta Q_{N, T}^{(\epsilon)}} .
\end{aligned}
$$


Next,

$$
\begin{aligned}
I_{2} & \leq \frac{C \epsilon}{\delta Q_{N, T}^{(\epsilon)}} \sum_{k=1}^{N}\left\{\frac{v_{k}^{2}}{\lambda_{k}+1} \bar{e}^{-2\left(\lambda_{k}-\theta\right) T} \int_{0}^{T} e^{2\left(\lambda_{k}-\theta\right) s} d s\right\}^{1 / 2} \\
& \leq \frac{C \epsilon}{\delta Q_{N, T}^{(\epsilon)}} \sum_{k=1}^{N}\left\{\frac{v_{k}^{2}}{\lambda_{k}+1} \frac{1}{2\left(\lambda_{k}-\theta\right)}\left(1-\bar{e}^{-2 T\left(\lambda_{k}-\theta\right)}\right)\right\}^{1 / 2} \\
& \leq \frac{C \epsilon}{\delta Q_{N, T}^{(\epsilon)}} \sum_{k=1}^{N}\left\{\frac{v_{k}^{2}}{\left(\lambda_{k}+1\right)\left(\lambda_{k}-\theta\right)}\right\}^{1 / 2} \\
& \leq \frac{C \epsilon}{\delta Q_{N, T}^{(\epsilon)}} \sum_{k=1}^{N} \frac{\|f\|}{k^{2}} \leq \frac{C \epsilon}{\delta Q_{N, T}^{(\epsilon)}} .
\end{aligned}
$$

In addition,

$$
\begin{aligned}
I_{3} & \leq \frac{C \epsilon^{2}}{\delta Q_{N, T}^{(\epsilon)}} \sum_{k=1}^{N}\left\{\frac{1}{\left(\lambda_{k}-\theta\right)^{2}} e^{4\left(\lambda_{k}-\theta\right) T} E\left[\int_{0}^{T} e^{\left(\lambda_{k}-\theta\right) s} d W_{k}(s)\right]^{4}\right\}^{1 / 2} \\
& \leq \frac{C \epsilon^{2}}{\delta Q_{N, T}^{(\epsilon)}} \sum_{k=1}^{N}\left\{\frac{1}{\left(\lambda_{k}-\theta\right)^{2}} \frac{\bar{e}^{4\left(\lambda_{k}-\theta\right) T}}{4\left(\lambda_{k}-\theta\right)^{2}}\left[e^{2\left(\lambda_{k}-\theta\right) T}-1\right]^{2}\right\}^{1 / 2} \\
& =\frac{C \epsilon^{2}}{\delta Q_{N, T}^{(\epsilon)}} \sum_{k=1}^{N}\left\{\frac{1}{\left(\lambda_{k}-\theta\right)^{2}} e^{-2\left(\lambda_{k}-\theta\right) T}\left[e^{2\left(\lambda_{k}-\theta\right) T}-1\right]\right\} \\
& =\frac{C \epsilon^{2}}{\delta Q_{N, T}^{(\epsilon)}} \sum_{k=1}^{N}\left\{\frac{1}{\left(\lambda_{k}-\theta\right)^{2}}\left(1-\bar{e}^{-2\left(\lambda_{k}-\theta\right) T}\right)\right\} \\
& \leq \frac{C \epsilon^{2}}{\delta Q_{N, T}^{(\epsilon)}} \sum_{k=1}^{N} \frac{1}{\left(\lambda_{k}-\theta\right)^{2}} \leq \frac{C \epsilon^{2}}{\delta Q_{N, T}^{(\epsilon)}} \sum_{k=1}^{N} \frac{1}{k^{4}} \\
& \leq \frac{C \epsilon^{2}}{\delta Q_{N, T}^{(\epsilon)}} .
\end{aligned}
$$

Note that

$$
\begin{aligned}
Q_{N, T}^{(\epsilon)} & =\sum_{k=1}^{N} \frac{\lambda_{k}+1}{2\left(\theta-\lambda_{k}\right)}\left\{v_{k}^{2}\left(e^{2\left(\theta-\lambda_{k}\right) T}-1\right)-\frac{\epsilon^{2} T}{\lambda_{k}+1}\right\} \\
& =\sum_{k=1}^{N} \frac{\lambda_{k}+1}{2\left(\lambda_{k}-\theta\right)}\left\{v_{k}^{2}\left(1-\bar{e}^{2\left(\lambda_{k}-\theta\right) T}\right)+\frac{\epsilon^{2} T}{\lambda_{k}+1}\right\} .
\end{aligned}
$$

Using (3.18), (3.19), and (3.20), we get that

$$
I_{1}+I_{2}+I_{3} \leq \frac{C_{1} \epsilon}{\delta Q_{N, T}^{(\epsilon)}}+\frac{C_{2} \epsilon T^{1 / 2}}{\delta Q_{N, T}^{(\epsilon)}}+\frac{C_{3} \epsilon^{2}}{\delta Q_{N, T}^{(\epsilon)}} \leq \frac{C \epsilon}{\delta Q_{N, T}^{(\epsilon)}}\left(1+T^{1 / 2}\right) .
$$

This completes the proof of Theorem 3.2. 
Observe that

$$
Q_{N, T}^{(\epsilon)} \geq C\left[\epsilon^{2} T+\|f\|^{2}\right]
$$

for large $N \geq N_{0}$ depending on $\theta$ and $T$ and for all $0<\epsilon<1$. Choosing $\delta=\epsilon^{1-r}$, for some $0<r<1$, we get that the bound in Theorem 3.2 is of order

$$
\frac{C \epsilon^{r}\left(1+T^{1 / 2}\right)}{\left(\epsilon^{2} T+\|f\|^{2}\right)}
$$

As a consequence of Theorems 3.1 and 3.2, we have the following main result giving a Berry-Esseen type bound for the MLE $\hat{\theta}_{N, \epsilon}$.

Theorem 3.5. Let $N \geq N_{0}$ be fixed, satisfying (3.23). Then there exists a constant $C$ depending on $\theta_{0},\|f\|$, and $T$ such that, for any $0<\epsilon<1$ and $0<r<1$,

$$
\sup _{y}\left|P_{\theta_{0}}^{\epsilon, N}\left\{\sqrt{Q_{N, T}^{(\epsilon)}} \epsilon^{-1}\left(\hat{\theta}_{N, \epsilon}-\theta_{0}\right) \leq y\right\}-\Phi(y)\right| \leq \frac{C \epsilon^{r}\left(1+T^{1 / 2}\right)}{\epsilon^{2} T+\|f\|^{2}}+3 \sqrt{\epsilon^{1-r}} .
$$

Remarks 3.6. Observe that the bound in Theorem 3.5 is of order $O\left(\epsilon^{r}\right)+O\left(\epsilon^{(1-r) / 2}\right)$. Choosing $r=1 / 3$, we note that the bound is of order $O\left(\epsilon^{1 / 3}\right)$.

\section{SPDE with linear drift (singular case): estimation and Berry-Esseen type bound}

Let $(\Omega, \mathscr{F}, P)$ be a probability space and consider the process $u_{i \epsilon}(t, x), 0 \leq x \leq 1,0 \leq t \leq T$, governed by the SPDE

$$
d u_{\epsilon}(t, x)=\theta \Delta u_{\epsilon}(t, x) d t+\epsilon(I-\Delta)^{-1 / 2} d W(t, x),
$$

where $\theta>0$ satisfies the initial and boundary conditions

$$
\begin{gathered}
u_{\epsilon}(0, x)=f(x), \quad 0<x<1, f \in L_{2}[0,1] \\
u_{\epsilon}(t, 0)=u_{\epsilon}(t, 1)=0, \quad 0 \leq t \leq T .
\end{gathered}
$$

Here, $I$ is the identity operator, $\Delta=\partial^{2} / \partial x^{2}$ as defined in Section 3, and the process $W(t, x)$ is the cylindrical Brownian motion in $L_{2}[0,1]$. In analogy with the discussion following the stochastic differential equation given by (2.6), it can be checked that the Fourier coefficients $u_{i \epsilon}(t)$ satisfy the stochastic differential equation

$$
d u_{i \epsilon}(t)=-\theta \lambda_{i} u_{i \epsilon}(t) d t+\frac{\epsilon}{\sqrt{\lambda_{1}+1}} d W_{i}(t), \quad 0<t \leq T,
$$

where conditions (2.7) hold.

Let $P_{\theta}^{\epsilon}$ be the measure generated by the process $u_{\epsilon}$ on $C[0, T]$ when $\theta$ is the true parameter. It can be shown that the family of measures $\left\{P_{\theta}^{\epsilon}, \theta \in \Theta\right\}$ does not form a family of equivalent probability measures. In fact, $P_{\theta}^{\epsilon}$ is singular with respect to $P_{\theta^{\prime}}^{\epsilon}$ when $\theta \neq \theta^{\prime}$ in $\Theta$ (cf. Hübner et al. [3]). Let $u_{\epsilon}^{(N)}(t, x)$ be the projection of $u_{\epsilon}(t, x)$ onto the subspace 
spanned by $\left\{e_{1}, e_{2}, \ldots, e_{N}\right\}$ in $L_{2}[0,1]$. In other words,

$$
u_{\epsilon}^{(N)}(t, x)=\sum_{i=1}^{N} u_{i \epsilon}(t) e_{i}(x)
$$

Let $P_{\theta}^{\epsilon, N}$ be the probability measure generated by the process $u_{\epsilon}^{(N)}$ on the subspace spanned by $\left\{e_{1}, \ldots, e_{N}\right\}$ in $L_{2}[0,1]$. It can be shown that the measures $\left\{P_{\theta}^{\epsilon, N}, \theta \in \Theta\right\}$ form an equivalent family and

$$
\begin{aligned}
\log \frac{d P_{\theta}^{\epsilon, N}}{d P_{\theta_{0}}^{(\epsilon, N)}}\left(u_{\epsilon}^{(N)}\right)=-\frac{1}{\epsilon^{2}} \sum_{i=1}^{N} \lambda_{i}\left(\lambda_{i}+1\right)[ & \left(\theta-\theta_{0}\right) \int_{0}^{T} u_{i \epsilon}(t)\left(d u_{i \epsilon}(t)+\theta_{0} \lambda_{i} u_{i \epsilon}(t) d t\right) \\
& \left.+\frac{1}{2}\left(\theta-\theta_{0}\right)^{2} \lambda_{i} \int_{0}^{T} u_{i \epsilon}^{2}(t) d t\right] .
\end{aligned}
$$

It can be checked that the MLE $\hat{\theta}_{N, \epsilon}$ of $\theta$ based on $u_{\epsilon}^{(N)}$ satisfies the likelihood equation

$$
\alpha_{\epsilon, N}=\epsilon^{-1}\left(\hat{\theta}_{N, \epsilon}-\theta_{0}\right) \beta_{\epsilon, N}
$$

when $\theta_{0}$ is the true parameter,

$$
\begin{aligned}
& \alpha_{\epsilon, N}=\sum_{i=1}^{N} \lambda_{i} \sqrt{\lambda_{i}+1} \int_{0}^{T} u_{i \epsilon}(t) d W_{i}(t), \\
& \beta_{\epsilon, N}=\sum_{i=1}^{N}\left(\lambda_{i}+1\right) \lambda_{i}^{2} \int_{0}^{T} u_{i, \epsilon}^{2}(t) d t .
\end{aligned}
$$

From (4.6), we obtain that

$$
\sqrt{R_{N, T}^{(\epsilon)}}\left(\hat{\theta}_{N, \epsilon}-\theta_{0}\right)=\frac{\epsilon\left\{\sum_{i=1}^{N} \lambda_{i} \sqrt{\lambda_{i}+1} \int_{0}^{T} u_{i \epsilon}(t) d W_{i}(t)\right\} / \sqrt{R_{N, T}^{(\epsilon)}}}{\left\{\sum_{i=1}^{N}\left(\lambda_{i}+1\right) \lambda_{i}^{2} \int_{0}^{T} u_{i \epsilon}^{2}(t) d t\right\} / R_{N, T}^{(\epsilon)}},
$$

where

$$
R_{N, T}^{(\epsilon)}=\sum_{i=1}^{N} \frac{\lambda_{i}\left(\lambda_{i}+1\right)}{2 \theta}\left\{v_{i}^{2}\left(1-\bar{e}^{-2 \theta \lambda_{i} T}\right)+T \frac{\epsilon^{2}}{\lambda_{i}+1}\right\} .
$$

It can be checked that

$$
E_{\theta_{0}} \int_{0}^{T} u_{i \epsilon}^{2}(t) d t<\infty
$$


118 Berry-Esseen type bound for SPDE

Theorem 4.1. For any $0<\delta<1$,

$$
\begin{aligned}
\sup _{y} \mid & P_{\theta_{0}}^{\epsilon, N}\left\{\sqrt{R_{N, T}^{(\epsilon)}}\left(\hat{\theta}_{N, \epsilon}-\theta_{0}\right) \leq y\right\}-\Phi(y) \mid \\
& \leq 2 P_{\theta_{0}}^{\epsilon, N}\left\{\left|\frac{\epsilon^{-1} \sum_{i=1}^{N}\left(\lambda_{i}+1\right) \lambda_{i}^{2} \int_{0}^{T} u_{i \epsilon}^{2}(t) d t}{R_{N, T}^{(\epsilon)}}-1\right| \geq \delta\right\}+3 \sqrt{\delta} .
\end{aligned}
$$

We can prove Theorem 4.1 using Lemmas 3.3 and 3.4 and following the method in the proof of Theorem 3.1.

Theorem 4.2. Let $0<\epsilon<1$ be fixed. Then there exists a constant $C$ depending on $\theta_{0},\|f\|^{2}$, and $T$ such that, for any $0<\delta<1$ and $N \geq 1$,

$$
P_{\theta_{0}}^{\epsilon, N}\left\{\left|\frac{\epsilon^{-1} \sum_{i=1}^{N}\left(\lambda_{i}+1\right) \lambda_{i}^{2} \int_{0}^{T} u_{i \epsilon}^{2}(t) d t}{R_{N, T}^{(\epsilon)}}-1\right| \geq \delta\right\} \leq \frac{C N^{3}\left(1+T^{1 / 2}\right)}{\delta R_{N, T}^{(\epsilon)}} .
$$

Proof. By the Îto formula, we get that

$$
d\left(u_{i \epsilon}^{2}(t)\right)=-2 \theta \lambda_{i} u_{i \epsilon}^{2}(t) d t+\frac{\epsilon}{\sqrt{\lambda_{i}+1}} u_{i \epsilon}(t) d W_{i}(t)+\frac{\epsilon^{2}}{\lambda_{i}+1} d t
$$

or, equivalently,

$$
d\left(\frac{\lambda_{i}\left(\lambda_{i}+1\right)}{2 \theta} u_{i \epsilon}^{2}(t)\right)=-\lambda_{i}^{2}\left(\lambda_{i}+1\right) u_{i \epsilon}^{2}(t) d t+\frac{\epsilon \lambda_{i} \sqrt{\lambda_{i}+1}}{2 \theta} u_{i \epsilon}(t) d W_{i}(t)+\frac{\epsilon^{2} \lambda_{i}}{2 \theta} d t
$$

or

$$
\begin{aligned}
& \frac{\lambda_{i}\left(\lambda_{i}+1\right)}{2 \theta} u_{i \epsilon}^{2}(T)-\frac{\lambda_{i}\left(\lambda_{i}+1\right)}{2 \theta} v_{i}^{2} \\
& \quad=-\int_{0}^{T} \lambda_{i}^{2}\left(\lambda_{i}+1\right) u_{i \epsilon}^{2}(t) d t+\frac{\epsilon \lambda_{i} \sqrt{\lambda_{i}+1}}{2 \theta} \int_{0}^{T} u_{i \epsilon}(t) d W_{i}(t)+\frac{\epsilon^{2} \lambda_{i}}{2 \theta} T .
\end{aligned}
$$

Again, by the Îto formula, it follows that

$$
\begin{aligned}
d\left(u_{i \epsilon}(t) e^{\theta \lambda_{i} t}\right)= & \frac{\epsilon}{\sqrt{\lambda_{i}+1}} e^{\theta \lambda_{i} t} d W_{i}(t), \\
u_{i \epsilon}(T) e^{\theta \lambda_{i} T}-v_{i}= & \int_{0}^{T} \frac{\epsilon}{\sqrt{\lambda_{i}+1}} e^{\theta \lambda_{i} t} d W_{i}(t), \\
u_{i \epsilon}(T)-v_{i} e^{\theta \lambda_{i} T}= & e^{\theta \lambda_{i} T} \int_{0}^{T} \frac{\epsilon}{\sqrt{\lambda_{i}+1}} e^{\theta \lambda_{i} t} d W_{i}(t), \\
u_{i \epsilon}^{2}(T)= & \bar{e}^{2 \theta \lambda_{i} T}\left(\int_{0}^{T} \frac{\epsilon}{\sqrt{\lambda_{i}+1}} e^{\theta \lambda_{i} t} d W_{i}(t)\right)^{2} \\
& +v_{i}^{2} \bar{e}^{2 \theta \lambda_{i} T}+\frac{2 \epsilon}{\sqrt{\lambda_{i}+1}} v_{i} \bar{e}^{-2 \theta \lambda_{i} T} \int_{0}^{T} e^{\theta \lambda_{i} t} d W_{i}(t),
\end{aligned}
$$


or

$$
\begin{aligned}
\frac{\lambda_{i}\left(\lambda_{i}+1\right)}{2 \theta} u_{i \epsilon}^{2}(T)= & \frac{\epsilon^{2} \lambda_{i}}{2 \theta} \bar{e}^{2 \theta \lambda_{i} T}\left(\int_{0}^{T} e^{\theta \lambda_{i} t} d W_{i}(t)\right)^{2} \\
& +\frac{\lambda_{i}\left(\lambda_{i}+1\right)}{2 \theta} v_{i}^{2} e^{-2 \theta \lambda_{i} T}+\frac{\epsilon}{\theta} \lambda_{i} \sqrt{\lambda_{i}+1} \bar{e}^{-2 \theta \lambda_{i} T} v_{i} \int_{0}^{T} e^{\theta \lambda_{i} t} d W_{i}(t) .
\end{aligned}
$$

From (4.16) and (4.18), we get that

$$
\begin{aligned}
& \sum_{i=1}^{N} \frac{\lambda_{i}\left(\lambda_{i}+1\right)}{2 \theta}\left\{v_{i}^{2}\left(1-\bar{e}^{2 \theta \lambda_{i} T}\right)+\frac{\epsilon^{2}}{\lambda_{i}+1} T\right\}-\sum_{i=1}^{N} \int_{0}^{T} \lambda_{i}^{2}\left(\lambda_{i}+1\right) u_{i \epsilon}^{2}(t) d t \\
& =\frac{\epsilon^{2}}{2 \theta} \sum_{i=1}^{N} \lambda_{i} \bar{e}^{2 \theta \lambda_{i} T}\left(\int_{0}^{T} e^{\theta \lambda_{i} t} d W_{i}(t)\right)^{2}+2 \epsilon \sum_{i=1}^{N} \lambda_{i} \sqrt{\lambda_{i}+1} v_{i} \bar{e}^{-2 \theta \lambda_{i} T} \int_{0}^{T} e^{\theta \lambda_{i} t} d W_{i}(t) \\
& \quad-\epsilon \sum_{i=1}^{N} \frac{\lambda_{i} \sqrt{\lambda_{i}+1}}{2 \theta} \int_{0}^{T} u_{i \epsilon}(t) d W_{i}(t) .
\end{aligned}
$$

Hence

$$
\begin{aligned}
P_{\theta_{0}}^{\epsilon, N}\{\mid & \left.\frac{\epsilon^{-1} \sum_{i=1}^{N} \int_{0}^{T} \lambda_{i}^{2}\left(\lambda_{i}+1\right) u_{i \epsilon}^{2}(t) d t}{R_{N, T}^{(\epsilon)}}-1 \mid \geq \delta\right\} \\
\leq & P_{\theta_{0}}^{\epsilon, N}\left\{\left|\frac{(\epsilon / 2 \theta) \sum_{i=1}^{N} \lambda_{i} e^{-2 \theta \lambda_{i} T}\left(\int_{0}^{T} e^{\theta \lambda_{i} t} d W_{i}(t)\right)^{2}}{R_{N, T}^{(\epsilon)}}\right| \geq \frac{\delta}{3}\right\} \\
& +P_{\theta_{0}}^{\epsilon, N}\left\{\left|\frac{(1 / \theta) \sum_{i=1}^{N} \lambda_{i} \sqrt{\lambda_{i}+1} v_{i} e^{-2 \theta \lambda_{i} T} \int_{0}^{T} e^{\theta \lambda_{i} t} d W_{i}(t)}{R_{N, T}^{(\epsilon)}}\right| \geq \frac{\delta}{3}\right\} \\
& +P_{\theta_{0}}^{\epsilon, N}\left\{\left|\frac{(1 / 2 \theta) \sum_{i=1}^{N} \lambda_{i} \sqrt{\lambda_{i}+1} \int_{0}^{T} u_{i \epsilon}(t) d W_{i}(t)}{R_{N, T}^{(\epsilon)}}\right| \geq \frac{\delta}{3}\right\} \\
= & J_{1}+J_{2}+J_{3} \text { (say), }
\end{aligned}
$$

where

$$
R_{N, T}^{(\epsilon)}=\sum_{i=1}^{N} \frac{\lambda_{i}\left(\lambda_{i}+1\right)}{2 \theta}\left\{v_{i}^{2}\left(1-\bar{e}^{-2 \theta \lambda_{i} T}\right)+\frac{\epsilon^{2}}{\lambda_{i}+1} T\right\} .
$$

Therefore,

$$
\begin{aligned}
J_{1} & \leq \frac{C \epsilon}{\delta R_{N, T}^{(\epsilon)}} \sum_{i=1}^{N} \lambda_{i} \bar{e}^{2 \theta \lambda_{i} T} \int_{0}^{T} e^{2 \theta \lambda_{i} t} d t \\
& =\frac{C \epsilon}{\delta R_{N, T}^{(\epsilon)}} \sum_{i=1}^{N} \lambda_{i} e^{-2 \theta \lambda_{i} T}\left\{\frac{\left(e^{2 \theta \lambda_{i} T}-1\right)}{2 \theta \lambda_{i}}\right\} \\
& \leq \frac{C \epsilon N}{\delta R_{N, T}^{(\epsilon)}},
\end{aligned}
$$




$$
\begin{aligned}
J_{2} & \leq \frac{C}{\delta R_{N, T}^{(\epsilon)}} \sum_{i=1}^{N} \sqrt{\lambda_{i}} \sqrt{\lambda_{i}+1} v_{i} \\
& \leq \frac{C N^{3}}{\delta R_{N, T}^{(\epsilon)}}, \\
J_{3} & \leq \frac{C}{\delta R_{N, T}^{(\epsilon)}}\left(\sum_{i=1}^{N} \lambda_{i}^{2}\left(\lambda_{i}+1\right) \int_{0}^{T} E u_{i \epsilon}^{2}(t) d t\right)^{1 / 2} \\
& \leq \frac{C \sqrt{2 \theta}}{\delta R_{N, T}^{(\epsilon)}}\left\{\sum_{i=1}^{N} \lambda_{i}\left(\lambda_{i}+1\right) v_{i}^{2}\left(1-\bar{e}^{-2 \theta \lambda_{i} T}\right)+T \sum_{i=1}^{N} \lambda_{i}\right\}^{1 / 2}
\end{aligned}
$$

(following [3, page 158])

$$
\begin{aligned}
& \leq \frac{C}{\delta R_{N, T}^{(\epsilon)}}\left\{\sum_{i=1}^{N} \lambda_{i}\left(\lambda_{i}+1\right)+T \sum_{i=1}^{N} \lambda_{i}\right\}^{1 / 2} \\
& \leq \frac{C}{\delta R_{N, T}^{(\epsilon)}}\left\{N^{5 / 2}+T^{1 / 2} N^{3 / 2}\right\} .
\end{aligned}
$$

Hence

$$
J_{1}+J_{2}+J_{3} \leq \frac{C}{\delta R_{N, T}^{(\epsilon)}}\left(N^{3}+T^{1 / 2} N^{3 / 2}\right) \leq \frac{C}{\delta R_{N, T}^{(\epsilon)}} N^{3}\left(1+T^{1 / 2}\right)
$$

This completes the proof of Theorem 4.2.

Observe that

$$
\begin{aligned}
R_{N, T}^{(\epsilon)} & =\sum_{k=1}^{N} \frac{\lambda_{k}\left(\lambda_{k}+1\right)}{2 \theta}\left\{v_{k}^{2}\left(1-e^{-2 \theta \lambda_{k} T}\right)+\frac{T \epsilon^{2}}{\lambda_{k}+1}\right\} \\
& \geq C \sum_{k=k_{1}}^{N} k^{4}\left\{v_{k}^{2}+\frac{T \epsilon^{2}}{k^{2}}\right\} \\
& \geq C\left(\sum_{k=1}^{N} k^{4} v_{k}^{2}+\epsilon^{2} T N^{3}\right)
\end{aligned}
$$

for some $k_{1}$ depending on $\epsilon, \theta$, and $T$, and hence for $N \geq N_{0}$ depending on $\epsilon, \theta$, and $T$. Therefore,

$$
J_{1}+J_{2}+J_{3} \leq \frac{C N^{3}\left(1+T^{1 / 2}\right)}{\delta\left(\epsilon^{2} T N^{3}+\sum_{k=1}^{N} k^{4} v_{k}^{2}\right)}
$$


for $N \geq N_{0}$ depending on $\epsilon, \theta$, and $T$. Choosing $\delta=N^{-\gamma}$, for some $\gamma>0$, we get that the bound is of order

$$
\frac{C N^{3}\left(1+T^{1 / 2}\right)}{N^{-\gamma}\left(\epsilon^{2} T N^{3}+\sum_{k=1}^{N} k^{4} v_{k}^{2}\right)} .
$$

As a consequence of Theorems 4.1 and 4.2, we have the following result which gives a Berry-Esseen type bound for the MLE $\hat{\theta}_{N, \epsilon}$ for any fixed $0<\epsilon<1$.

Theorem 4.3. Let $0<\epsilon<1$ be fixed. Then there exists a constant $C$ depending on $\theta_{0},\|f\|^{2}$, and $T$ such that, for any $\gamma>0$ and $N \geq N_{0}$, depending on $\epsilon, \theta_{0}$, and $T$,

$$
\begin{aligned}
\sup _{y} \mid P_{\theta_{0}}^{\epsilon, N} & \left\{\sqrt{R_{N, T}^{(\epsilon)}}\left(\hat{\theta}_{N, \epsilon}-\theta_{0}\right) \leq y\right\}-\Phi(y) \mid \\
& \leq \frac{C N^{3}}{N^{-\gamma}}\left(\frac{1+T^{1 / 2}}{\epsilon^{2} T N^{3}+\sum_{k=1}^{N} k^{4} v_{k}^{2}}\right)+3 \sqrt{N^{-\gamma}} .
\end{aligned}
$$

Remarks 4.4. Observe that the bound in Theorem 4.3 is of order $O\left(N^{\gamma-2}\right)+O\left(N^{-\gamma / 2}\right)$ provided $\sum_{k=1}^{N} k^{4} v_{k}^{2} \geq g(N)=O\left(N^{5}\right)$. In such a case, the bound can be obtained to be of order $O\left(N^{-2 / 3}\right)$ by choosing $\gamma=4 / 3$. We can obtain the rate of convergence for the case when $N$ is fixed but $\epsilon$ varies over the interval $(0,1)$ by arguments similar to those given above. We omit the details.

\section{References}

[1] P. D. Feigin, Maximum likelihood estimation for continuous-time stochastic processes, Adv. in Appl. Probab. 8 (1976), no. 4, 712-736.

[2] P. Hall and C. C. Heyde, Martingale Limit Theory and Its Application, Academic Press, New York, 1980.

[3] M. Hübner, R. Khasminskii, and B. L. Rozovskii, Two examples of parameter estimation for stochastic partial differential equations, Stochastic Processes (S. Cambanis, J. K. Ghosh, R. L. Karandikar, and P. K. Sen, eds.), Springer-Verlag, New York, 1993, pp. 149-160.

[4] R. S. Liptser and A. N. Shiryayev, Statistics of Random Processes. II, Springer-Verlag, New York, 1978.

[5] R. Michel and J. Pfanzagl, The accuracy of the normal approximation for minimum contrast estimates, Z. Wahrscheinlichkeitstheorie und Verw. Gebiete 18 (1971), 73-84.

[6] M. N. Mishra and B. L. S. Prakasa Rao, On the Berry-Esseen bound for maximum likelihood estimator for linear homogeneous diffusion processes, Sankhyā Ser. A 47 (1985), no. 3, 392398.

[7] B. L. S. Prakasa Rao, Statistical inference for stochastic partial differential equations, Selected Proceedings of the Symposium on Inference for Stochastic Processes (Athens, Ga, 2000) (I. V. Basawa, C. C. Heyde, and R. L. Taylor, eds.), IMS Lecture Notes Monogr. Ser., vol. 37, Inst. Math. Statist., Ohio, 2001, pp. 47-70.

[8]_- On some problems of estimation for some stochastic partial differential equations, Uncertainty and Optimality (J. C. Misra, ed.), World Scientific Publishing, New Jersey, 2002, pp. 71-153. 


\section{Berry-Esseen type bound for SPDE}

[9] B. L. Rozovski1̌, Stochastic Evolution Systems. Linear Theory and Applications to Nonlinear Filtering, Mathematics and Its Applications (Soviet Series), vol. 35, Kluwer Academic Publishers, Dordrecht, 1990.

M. N. Mishra: Institute of Mathematics and Applications, Bhubaneswar 751013, India E-mail address: mnmeeshraw@yahoo.com

B. L. S. Prakasa Rao: Indian Statistical Institute, New Delhi 110016, India

E-mail address: blsp@isid.ac.in 


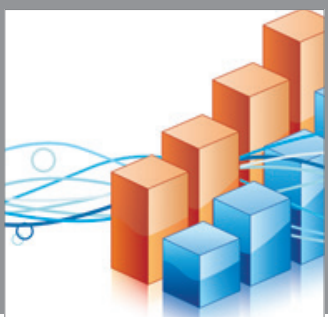

Advances in

Operations Research

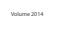

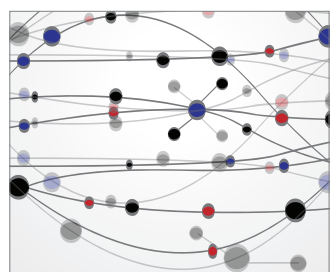

\section{The Scientific} World Journal
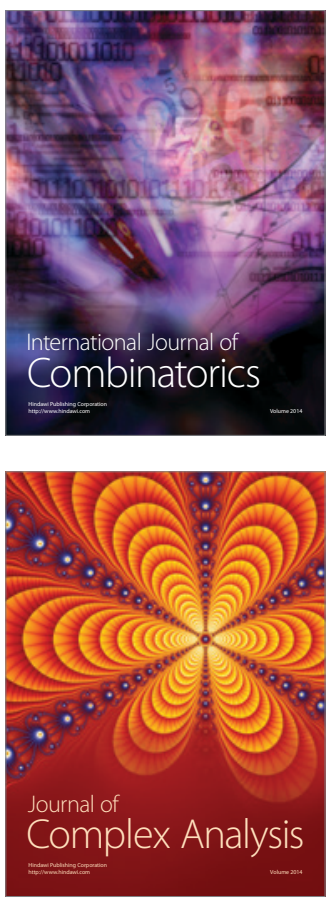

International Journal of

Mathematics and

Mathematical

Sciences
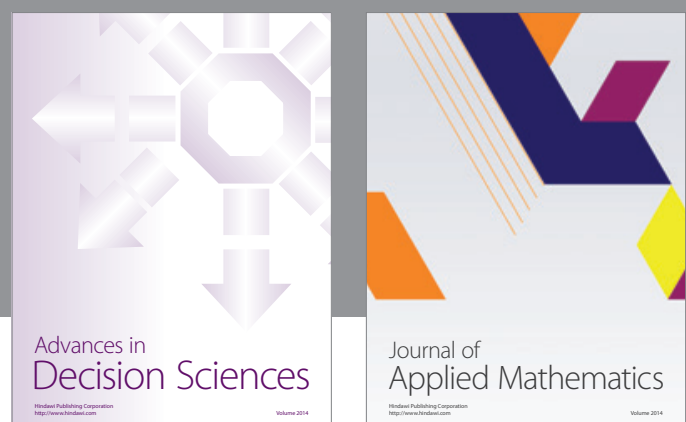

Journal of

Applied Mathematics
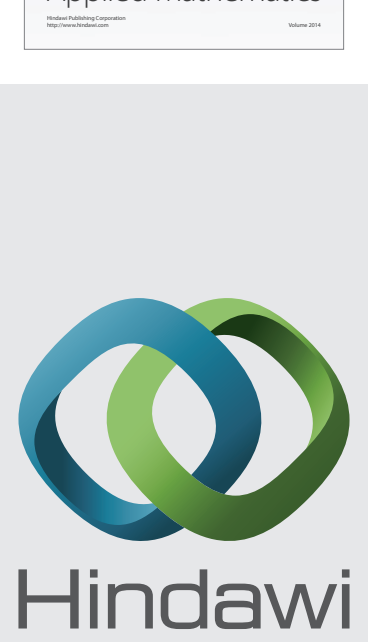

Submit your manuscripts at http://www.hindawi.com
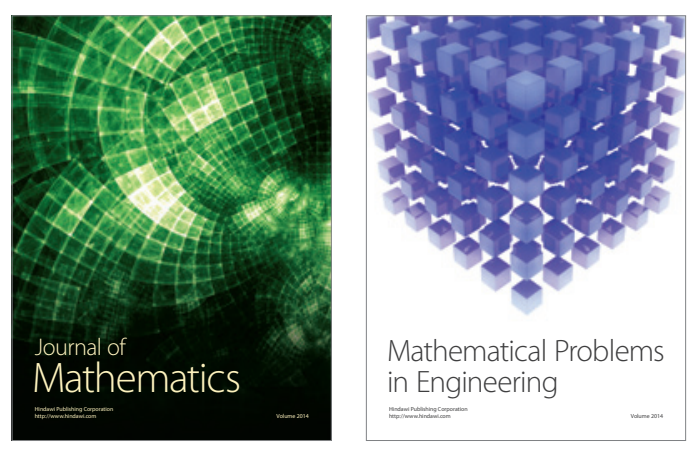

Mathematical Problems in Engineering
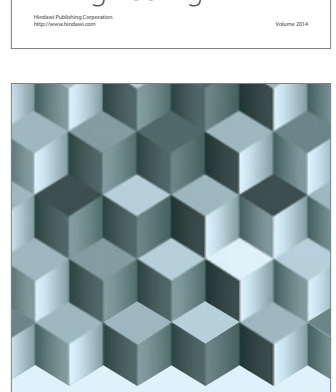

Journal of

Function Spaces
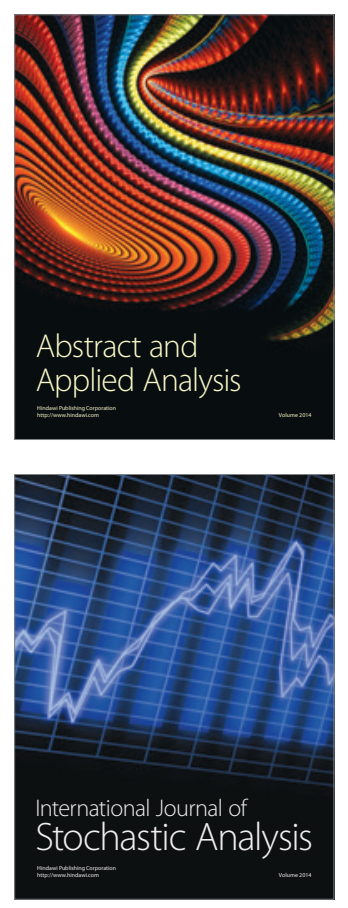

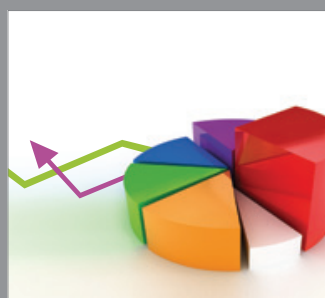

ournal of

Probability and Statistics

Promensencen
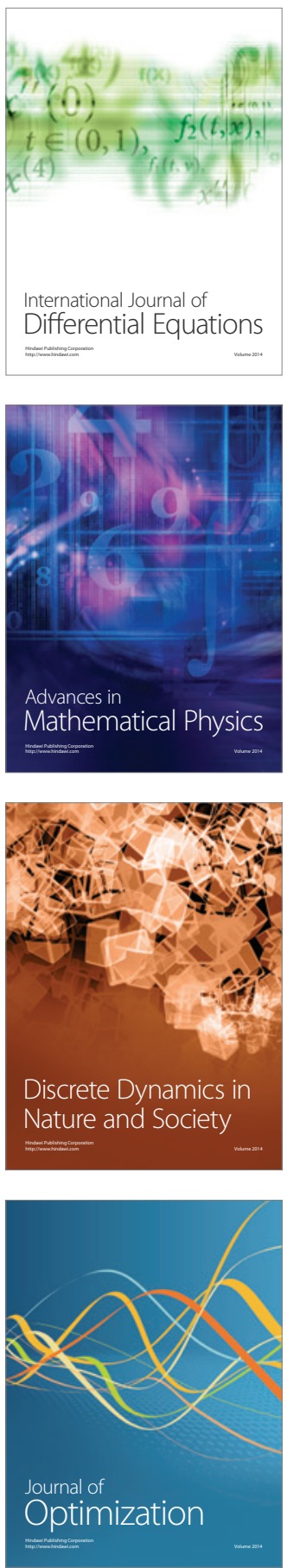